\title{
UNA ESPECIE NUEVA DE ECHEVERIA (CRASSULACEAE) ORIGINARIA DEL ESTADO DE JALISCO, MÉXICO*
}

\author{
Ignacio García Ruiz ${ }^{1, * *}$ y Emmanuel Pérez-CaliX ${ }^{2}$ \\ ${ }^{1}$ Instituto Politécnico Nacional, Centro Interdisciplinario de Investigación \\ para el Desarrollo Integral Regional, Unidad Michoacán \\ Justo Sierra 28, 59510 Jiquilpan, Michoacán, México \\ igarciar@ipn.mx \\ ${ }^{2}$ Instituto de Ecología, A.C., Centro Regional del Bajío, Apdo. postal 386 \\ 61600 Pátzcuaro, Michoacán, México.emmanuel.perezcalix@inecol.edu.mx
}

\section{RESUMEN}

Con base en ejemplares botánicos originarios del estado de Jalisco, México, se propone como especie nueva para la ciencia, describe e ilustra a Echeveria patriotica (Crassulaceae). Este taxon por llevar tallo, láminas foliares angostas en la base en forma de pseudopeciolo, inflorescencia paniculiforme con raquis alargado, con más de tres ramificaciones y corola cilíndrico-urceolada, de más de $13 \mathrm{~mm}$ de largo, se ubica en la serie Gibbiflorae (Baker) A. Berger. Del conjunto de especies involucradas en este grupo tiene más semejanza con $E$. grisea E. Walther, de la que difiere en el largo de los pedicelos, del cáliz y de la corola, que son más grandes en la nueva especie; además, se diferencian en el color de la flor y de los nectarios.

Palabras clave: Crassulaceae, Echeveria, Gibbiflorae, Jalisco, México.

\begin{abstract}
Based on botanical specimens collected in the state of Jalisco, Mexico, Echeveria patriotica (Crassulaceae) is described and illustrated. Due to the possession of the following
\end{abstract}

\footnotetext{
* Trabajo realizado con apoyo económico del Instituto de Ecología, A.C. (cuenta 902-06), del Consejo Nacional de Ciencia y Tecnología y de la Comisión Nacional para el Conocimiento y Uso de la Biodiversidad.

** Becario de la Comisión de Operación y Fomento para las Actividades Académicas y de Estímulos al Desempeño de los Investigadores del Instituto Politécnico Nacional.
} 
features, it is assigned to series Gibbiflorae (Baker) A. Berger: stem present, leaf blades narrowed at the base in the form of a pseudopetiole, inflorescence with more than 3 ramifications and an elongated rachis, and corolla cylindrical-urceolate more than $13 \mathrm{~mm}$ long. Of the species belonging to this section, E. patriotica is most similar to E. grisea E. Walther, from which it differs by having longer a pedicel, calyx and corolla. In addition, it is distinguished by the color of the flower and the nectaries.

Key words: Crassulaceae, Echeveria, Gibbiflorae, Jalisco, Mexico.

Con el objetivo de complementar el conocimiento florístico del noroeste del estado de Michoacán y de la zona colindante de Jalisco, se han realizado una serie de exploraciones botánicas en la región. En uno de los recorridos se colectaron ejemplares de plantas del género Echeveria (Crassulaceae) en el sur de la Sierra del Tigre, en el municipio de Mazamitla, Jalisco. En los intentos por asignarles nombre se observó que coinciden con los miembros de la serie Gibbiflorae (Baker) Berger (sensu Walther, 1972); sin embargo, presentan caracteres que los distinguen de las especies previamente descritas. Por lo anterior se propone:

Echeveria patriotica I. García et Pérez-Calix, sp. nov. Fig. 1 y 2.

Herba perennis caulescens, caulis usque $20 \mathrm{~cm}$ longus, folia rosulam laxam 20-35 cm diametro formantia, laminae carnosae oblongo-obovatae usque spathulatae 6-18 cm longae, panicula $20-100 \mathrm{~cm}$ alta, pedicelli $0.7-1.0 \mathrm{~cm}$ longi, flos $1.8-2 \mathrm{~cm}$ longus, $1.4 \mathrm{~cm}$ diametro ad basem, calyx ad instar stellae, sepalis patentibus inaequalibus deltoideo-lanceolatis, corolla cylindrico-urceolata, segmentis lanceolatis, ad basem cremea, alibi aurantiaco-rubella usque purpureo-rubra, nectaria scarlatino-rubra.

Planta herbácea, perenne, glabra, solitaria, caulescente; tallo erecto, ocasionalmente ramificado, hasta de $20 \mathrm{~cm}$ de largo y de $1-2.5 \mathrm{~cm}$ de diámetro cerca de la base; hojas 9-13, dispuestas en espiral, formando una roseta laxa de 10-35 cm de diámetro, láminas carnosas, oblongo-obovadas a espatuladas, de 6-18 cm de largo por 3.5-11 cm de ancho y de 0.5-1.6 cm de grueso cerca de la base, ápice redondeado, cortamente mucronado, de color verde, en ocasiones con el contorno rojizo, completamente rojizas con la edad, la base se angosta para formar un pseudopeciolo de 2-4 cm de longitud, canaliculado dorsalmente; inflorescencias 1 a 3 por roseta, en forma de panícula de 20-100 cm de alto, incluyendo el pedúnculo de ca. $1 \mathrm{~cm}$ de diámetro 


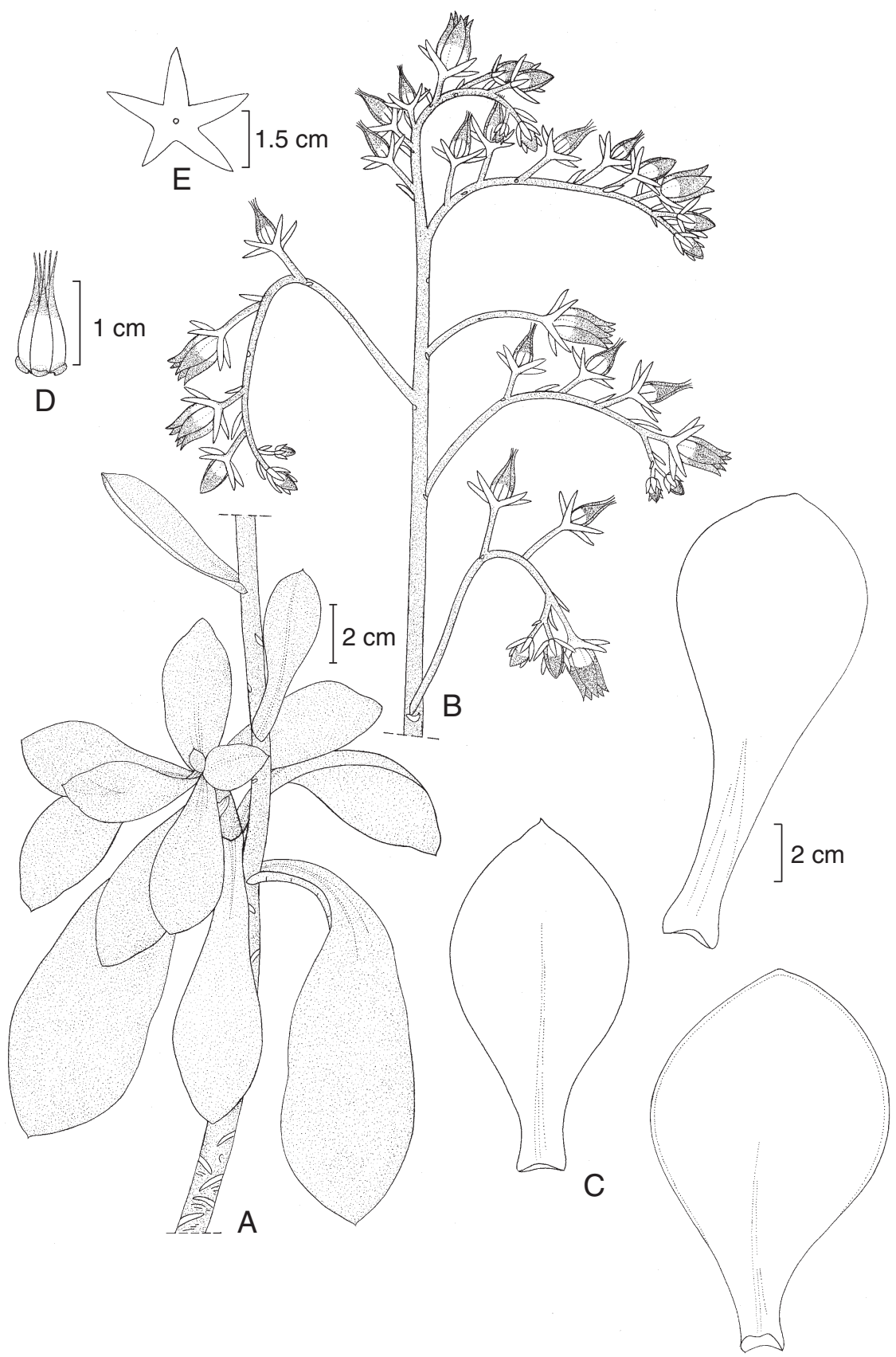

Fig. 1. Echeveria patriotica sp. nov. A. hábito de la planta; B. inflorescencia; C. variación de las láminas foliares; D. gineceo y nectarios; E. vista inferior del cáliz. Dibujo realizado por Rogelio Cárdenas Soriano a partir de I. García y Y. Hernández 4441. 
cerca de la base, con 1-7 ramas secundifloras con 1-8 flores cada una; brácteas dispuestas en espiral, adpresas, oblongo-lanceoladas, espatuladas u oblongo-obovadas, de $1.2-12 \mathrm{~cm}$ de largo, de $0.6-4 \mathrm{~cm}$ de ancho y de ca. $0.15 \mathrm{~cm}$ de grueso en la parte media y hasta de $0.85 \mathrm{~mm}$ de grueso cerca de la base, de color verde a rojizas con la edad, ápice redondeado, cortamente mucronado, base espolonada, deciduas; pedicelos de 0.7-1 cm de largo y ca. 0.25 de diámetro; flor de 1.8 a $2 \mathrm{~cm}$ de largo, de ca. $1.4 \mathrm{~cm}$ diámetro cerca de la base; cáliz en forma de estrella, sépalos fusionados en la base, segmentos extendidos, desiguales, deltoideo-lanceolados, de 0.9-1.4 cm de largo y de 0.3-0.6 cm de ancho; corola conoide en botón, cilíndrico-urceolada en la antesis, segmentos lanceolados, de $0.5-0.75 \mathrm{~cm}$ de ancho, fusionados en la base, formando un tubo de $0.1-0.15 \mathrm{~cm}$ de alto, segmentos con el ápice mucronado, rectos a ligeramente deflexos, con una costilla manifiesta en la parte media dorsal, de color blanco a blanco-crema desde la base hasta un poco abajo de la parte media, arriba hasta el ápice de color anaranjado-rojizo a rojo-púrpura; estambres 10, 5 opuestos a los pétalos, de $1.3 \mathrm{~cm}$ de largo, y 5 alternos de $1.5 \mathrm{~cm}$ de largo, anteras de $0.15-0.20$ $\mathrm{cm}$ de largo; nectarios reniformes, de color rojo-púrpura, de ca. $0.1 \mathrm{~cm}$ de largo y ca. $0.3 \mathrm{~cm}$ de ancho; carpelos 5, libres, de $1.2-1.5 \mathrm{~cm}$ de alto y $0.5-0.6 \mathrm{~cm}$ de ancho, estilos filiformes, de 0.8-0.9 cm de largo, de color rojo-púrpura; folículos erectos, de 1.2-1.8 cm de largo; semillas numerosas, de color café.

Tipo: México. Jalisco, municipio de Mazamitla, Plan de Cervantes, km 14.5 carretera del entronque Quitupan-Valle de Juárez, hacia Santa María del Oro; aproximadamente $5 \mathrm{~km}$ en línea recta al sur de la ranchería El Tigre. Alt. $2290 \mathrm{~m}$; lat. $19^{\circ} 51^{\prime} 15^{\prime \prime} \mathrm{N}$; long. 102 $566^{\prime} 50^{\prime \prime} \mathrm{W}$. Bosque de pino-encino (Pinus devoniana-Quercus castanea). 23. XI. 1996. I. García y Y. Hernández 4441 (holotipo IEB, isotipos CIMI, ENCB, MEXU, MICH).

Material adicional examinado: Jalisco, municipio de Mazamitla, $\mathrm{km} 8$ carretera del entronque Quitupan-Valle de Juárez hacia Santa María del Oro. Alt. 2200 m, ladera este de la carretera, cerca de bosque de encino-pino, con Lippia sp., Montanoa sp. y Buddleia sp., I. García y G. Hernández 5010-bis (CIMI, IEB); ibid., I. García et al. 6262 (CIMI, IEB, MICH, UCR), 6268 (CIMI, IEB, MICH, UCR); ibid., I. García y E. Pérez 6821 (CIMI).

Fenología, habitat y distribución: Echeveria patriotica se ha observado en floración de octubre a enero. Aunque es una planta preferentemente rupícola, también crece ocasionalmente como epífita de algunos encinos (Quercus spp.). Sus poblacio- 
nes se establecen en el sotobosque, así como en los cortes y laderas sombreadas a la orilla de la carretera en las inmediaciones del bosque de Pinus devoniana, Pinus oocarpa y Quercus castanea. Crece en asociación con Eryngium gracile, Senecio sp., Donellsmithia sp., Lamourouxia sp., en altitudes entre 2200 y 2300 m. Se conoce únicamente de dos poblaciones distantes entre sí por unos $4 \mathrm{~km}$.

Etimología: El epíteto específico obedece a los colores de la flor: verde (cáliz), blanco y rojo (base y ápice de la corola respectivamente), aludiendo a los colores de la bandera de México (Fig. 2).

El nuevo taxon se ubica en la serie Gibbiflorae (Baker) A. Berger. Moran (1974), Kimnach (2003) y Meyrán y López (2003) presentan un concepto de este grupo que incluye también a los miembros de la serie Retusae E. Walther. Sin embargo, aquí preferimos usar la propuesta de Walther (1972), quien integra en el mismo conjunto a elementos que reúnen las siguientes características:

-plantas usualmente glabras, con tallo robusto y por lo común monopódico o acaulescentes;

-hojas de tamaño mediano a grande y frecuentemente con la base angosta en forma de pseudopeciolo acanalado en el haz y carinado en el envés;

-inflorescencias paniculiformes con el raquis alargado y con más de tres ramificaciones secundifloras;

-brácteas similares a las hojas pero más pequeñas;

-pedicelos de hasta $10 \mathrm{~mm}$ de largo;

-sépalos desiguales, ascendentes a extendidos, deltoides a lineares u oblanceolados, por lo común agudos;

-corola de $13 \mathrm{~mm}$ o más de largo, de forma cilindroide-urceolada a campanulada, pentagonal, de color rosa-rojizo, púrpura o amarillo, más o menos glauca y pruinosa;

-pétalos carinados, con una cavidad basal prominente en la cara interna;

-nectarios grandes, gruesos y truncados, de color rojo-púrpura, blanquecinoamarillento o blanco-crema;

-estilos de color blanquecino o rojo-púrpura a casi negros.

La serie se puede dividir en dos subconjuntos, uno conformado por las especies acaules o que portan un tallo corto, hojas aglomeradas en una roseta basal, láminas foliares escasamente pseudopecioladas y con el ápice agudo; aquí corresponden: E. cante Glass \& Mendoza-García, E. dactylifera E. Walther, E. subrigida Robinson \& Seaton (E. palmeri Rose) y con excepción de la última que lleva los nectarios de 


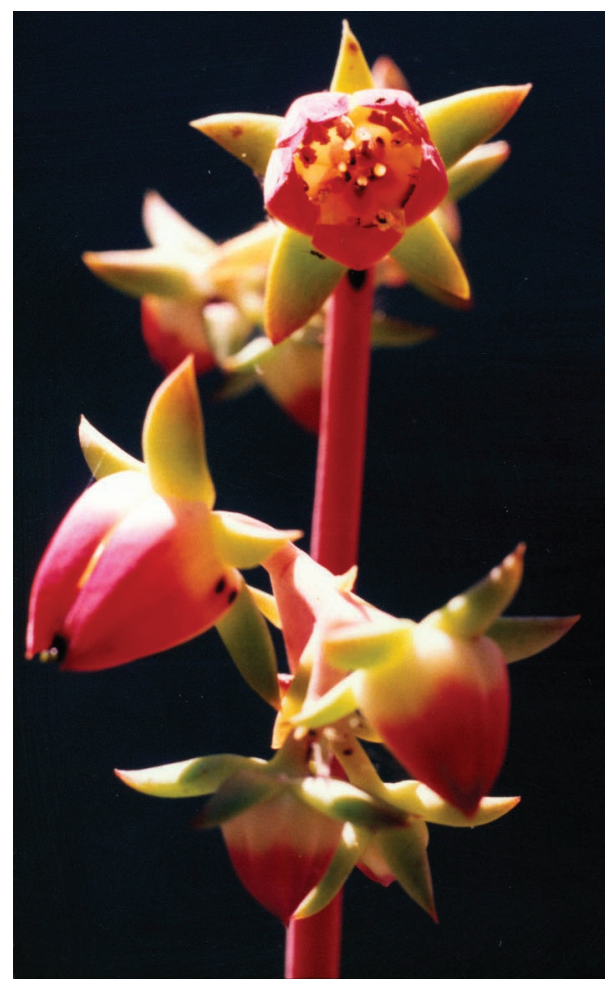

Fig. 2. Inflorescencia de Echeveria patriotica, las flores muestran los colores de la bandera mexicana que denotan el epíteto específico.

color rojo-púrpura, las otras los tienen blanquecino-amarillentos. El segundo subconjunto está integrado por plantas caulescentes, con pocas hojas, rara vez en roseta basal, las láminas por lo general son manifiestamente pseudopecioladas y sus flores tienen los nectarios pálidos; aquí pertenecen: E. acutifolia Lindley, E. crenulata Rose, E. fimbriata C. H. Thompson, E. gibbiflora De Candolle (E. grandifolia Haworth, E. violescens E. Walther, E. gibbiflora var. violescens (E. Walther) Kimnach), E. gigantea Rose \& Purpus, E. grisea E. Walther, E. longiflora E. Walther, E. pallida E. Walther, E. rubromarginata Rose y E. nayaritensis Kimnach.

De los taxa del primer subgrupo, Echeveria patriotica se asemeja a E. subrigida sólo por el color de los nectarios; pero, por su hábito y por la forma de las láminas foliares es más parecida a los del segundo. Aquí encuentra mayor similitud con E. grisea, de la que se diferencia en que el nuevo taxon tiene los pedicelos de 0.7 a $1 \mathrm{~cm}$ de largo, los segmentos del cáliz de 0.9 a $1.4 \mathrm{~cm}$ de largo, la corola de 1.8 a $2 \mathrm{~cm}$ de largo, en la corola la coloración es blanca a blanco-crema desde la base 
hasta un poco debajo de la parte media y de ahí hasta el ápice anaranjado-rojiza a rojo-púrpura y los nectarios son de color rojo-púrpura. Por su parte, E. grisea tiene pedicelos de $0.4 \mathrm{~cm}$ o menos de largo, los segmentos del cáliz miden menos de 0.7 $\mathrm{cm}$, la corola es de ca. $1.3 \mathrm{~cm}$ de largo, de color rosa y los nectarios son de color amarillo pálido (Cuadro 1).

Cuadro 1. Comparación morfológica de Echeveria patriotica y E. grisea.

\begin{tabular}{|c|c|c|}
\hline & E. patriotica & E. grisea \\
\hline $\begin{array}{l}\text { Hojas: } \\
\text { forma } \\
\text { largo }(\mathrm{cm}) \\
\text { ancho }(\mathrm{cm})\end{array}$ & $\begin{array}{l}\text { oblongo-obovada a espatulada } \\
6-18 \\
3.5-11\end{array}$ & $\begin{array}{l}\text { ampliamente obovado-espatulada } \\
10-15 \\
5-8\end{array}$ \\
\hline $\begin{array}{l}\text { Brácteas: } \\
\text { forma } \\
\text { largo }(\mathrm{cm}) \\
\text { ancho }(\mathrm{cm})\end{array}$ & $\begin{array}{l}\text { oblongo-lanceolada, espatulada u } \\
\text { oblongo-obovada } \\
1.2-12 \\
0.6-4.0\end{array}$ & $\begin{array}{l}\text { oblongo-obovada } \\
5 \\
2\end{array}$ \\
\hline $\begin{array}{l}\text { Pedicelo: } \\
\text { largo }(\mathrm{cm})\end{array}$ & $0.7-1$ & 0.4 o menos \\
\hline $\begin{array}{l}\text { Segmentos de cáliz } \\
\text { largo }(\mathrm{cm})\end{array}$ & $0.9-1.4$ & 0.7 (el mayor) \\
\hline $\begin{array}{l}\text { Corola } \\
\text { largo }(\mathrm{cm}) \\
\text { color }\end{array}$ & $\begin{array}{l}1.8-2 \\
\text { blanco a blanco-crema desde la } \\
\text { base hasta un poco debajo de } \\
\text { la parte media y de ahí hasta el } \\
\text { ápice anaranjado-rojizo a rojo- } \\
\text { púrpura en el ápice }\end{array}$ & $\begin{array}{l}1.3 \\
\text { rosa }\end{array}$ \\
\hline $\begin{array}{l}\text { Nectarios } \\
\text { color }\end{array}$ & rojo-púrpura & amarillo pálido \\
\hline $\begin{array}{l}\text { Estilos } \\
\text { longitud (mm) } \\
\text { color }\end{array}$ & $\begin{array}{l}8-9 \\
\text { rojo-púrpura }\end{array}$ & carmín \\
\hline Habitat & $\begin{array}{l}\text { bosque de Quercus y } \\
\text { Pinus-Quercus }\end{array}$ & bosque tropical caducifolio \\
\hline Fenología & octubre-enero & agosto-octubre \\
\hline Distribución & Jalisco & Michoacán, Guerrero \\
\hline
\end{tabular}




\section{AGRADECIMIENTOS}

Agradecemos al Dr. Jerzy Rzedowski por la elaboración de la diagnosis en latín; él, la Maestra Graciela Calderón de Rzedowski, el Dr. Sergio Zamudio y la Biól. Patricia Mayoral revisaron el manuscrito; Yolanda Hernández, Germán Hernández y J. A. Machuca nos apoyaron en el trabajo de campo y el Dr. Victor Steinmann en la traducción del resumen al inglés. El dibujo se debe a la pluma de Rogelio Cárdenas.

\section{LITERATURA CITADA}

Kimnach, M. 2003. Echeveria. In: Eggli, U. (ed.). Illustrated handbook of succulent plants: Crassulaceae. Springer-Verlag. Berlin. pp. 103-128.

Meyrán, J. y L. López. 2003. Las crasuláceas de México. Sociedad Mexicana de Cactología, A.C. México, D.F. 234 pp. +52 láminas con fotografías.

Moran, R. 1974. Division of the genus Echeveria into series. In: Jacobsen, H. (ed.). Lexicon of succulent plants. Blandford Press. Londres. pp. 184-186.

Walther, E. 1972. Echeveria. California Academy of Sciences. San Francisco. 426 pp.

Recibido en enero de 2006. Aceptado en octubre de 2006. 